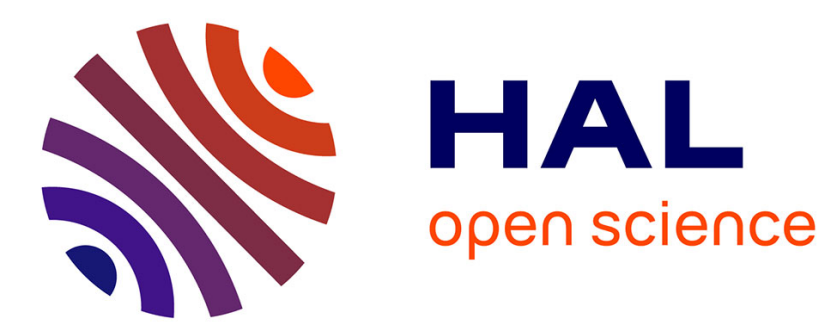

\title{
Plane anisotropic rari-constant materials
}

Paolo Vannucci, Boris Desmorat

\section{- To cite this version:}

Paolo Vannucci, Boris Desmorat. Plane anisotropic rari-constant materials. 2015. hal-01167906

\author{
HAL Id: hal-01167906 \\ https://hal.science/hal-01167906
}

Preprint submitted on 24 Jun 2015

HAL is a multi-disciplinary open access archive for the deposit and dissemination of scientific research documents, whether they are published or not. The documents may come from teaching and research institutions in France or abroad, or from public or private research centers.
L'archive ouverte pluridisciplinaire HAL, est destinée au dépôt et à la diffusion de documents scientifiques de niveau recherche, publiés ou non, émanant des établissements d'enseignement et de recherche français ou étrangers, des laboratoires publics ou privés. 


\title{
Plane anisotropic rari-constant materials
}

\author{
Paolo Vannucci ${ }^{1}$ \\ Université Paris-Saclay: LMV - Laboratoire de Mathématiques de Versailles \\ CNRS UMR8100 / Université Versailles St Quentin, Versailles - France. \\ Boris Desmorat \\ Institut J. Le Rond d'Alembert, CNRS UMR7190 / UPMC Univ Paris 6, Paris. \\ Université Paris-Sud 11, Orsay - France.
}

\begin{abstract}
We consider here the existence of rari-constant anisotropic layers, and show that actually there are two distinct classes of such materials, mutually exclusive. Also, we show that the correct condition for establishing that a material is of the rari-constant type is that the number of independent linear tensor invariants of the elastic tensors must reduce to one. We characterize these materials and show that they can be designed using some basic rules of homogenization.
\end{abstract}

\section{Introduction}

Within the standard paradigm of the hyper elastic continuum, we justify the existence of classes of plane anisotropy admitting four invariants instead of five. In particular, we show the existence of two mutually excluding cases.

Materials of this type have been widely studied in the past and their existence has been the subject of one of the most famous diatribes in the theory of elasticity: that between what Pearson [Todhunter and Pearson, 1886, p. 496] named multi-constant and rari-constant materials, [Benvenuto, 1991, v. 1, p. 227], [Dugas, 1950, p. 398], [Love, 1944, p. 6, p. 13].

The idea of rari-constant materials stems from the early works of Navier [1827] and his model of matter, known as molecular theory, first presented at Académie des Sciences on May 14, 1821. Basically, the model proposed by Navier aims at explaining the behavior of elastic solids as that of a lattice of particles (molecules) interacting together via central forces proportional to their mutual distance. This is not a new idea: it has its last foundation in the works of Newton [1687]. For what concerns the mechanics of solids,

\footnotetext{
${ }^{1}$ Corresponding author: Paolo Vannucci. LMV, 45 Avenue des Etats-Unis 75035 Versailles Cedex, Tel: +33 1392542 18, e-mail:paolo.vannucci@uvsq.fr
} 
the true initiator of the molecular theory is considered to be Boscovich [1743]; other works on this topic, before the mémoire of Navier, are those of Poisson [1811, 1814] on the equilibrium of bent plates, while subsequent fundamental contributions are due to Cauchy [1828a,b], still Poisson [1829] and de Saint-Venant [1844].

The basic idea in the classical molecular approach of Navier and Cauchy, the continuum as a limit of a discrete lattice of particles interacting together via central forces, has a direct consequence, Stackgold [1950], Doyle and Ericksen [1956]: 15 moduli describe the behavior of a completely anisotropic body in 3D, and only one modulus suffices to determine it for an isotropic material. These results was not confirmed by experimental tests, so doubts existed about its validity, until the molecular approach was completely bypassed by the theory proposed in 1837 by Green [1839]: no underlying microscopic structure of the matter, considered as a continuum, is assumed, and the basic property defining the elastic behavior is energetic: in non dissipative processes the internal forces derive from a quadratic potential.

The consequences of such an assumption lead to the multi-constant model: 21 independent moduli are necessary to describe the elastic response of a completely anisotropic body in 3D, which reduce to only 2 for an isotropic material. The results of the Green's theory were confirmed by experience which, together with its much simpler theoretical background, ensured the success of the multi-constant theory. Nonetheless, the diatribe between the molecular, rari-constant, and continuum, multi-constant, theories lasted a long period: which is the right number of elastic constants and the correct model of elastic continuum?

The further developments of the molecular model by Voigt [1887] and Poincaré [1892] are refined models that, enriching in different ways the original model of Navier, obtain multi-constant theories starting from a molecular model, see Ostoja-Starzewski [2007], Capecchi et al. [2011]. More recently, ideas inspired by the Navier-Cauchy approach has produced molecular dynamics models or models for explicating the behavior of complex bodies,

As an effect of this diatribe, the two models are usually considered as opposing and somewhat irreconcilable, though different researchers has made attempts to show that this is not the case, Born [1915], [Love, 1944, Note B, p. 616], [Muskhelishvili, 1953, p. 55].

We consider here the planar case and, using a continuum approach and the polar formalism, Verchery [1979], Vannucci [2005], we derive some simple conditions for the existence of rari-constant materials in terms of tensor invariants. In particular, we show that there exist two dual types that we name direct- and inverse- rari-constant materials and that the classical Cauchy-Poisson conditions for a continuum to be rari-constant are not sufficient to characterize such a material: the only true necessary and sufficient condition is the number of independent linear tensor invariants, that must 
be of one. We give also a simple example of how anisotropic rari-constant layers of both the types can be fabricated.

\section{Basic equations}

We consider the stiffness tensor $\mathbb{E}$ of an anisotropic elastic continuum; within the classical paradigm of elasticity, $\mathbb{E}$ possesses some symmetries for the indexes position (in the following named index symmetries):

- 45 minor symmetries, consequences of the symmetries of the stress and strain tensors: $\sigma=\sigma^{\top}, \varepsilon=\varepsilon^{\top} \Rightarrow$

$$
E_{i j k l}=E_{j i k l}=E_{i j l k}=E_{j i l k},
$$

that for the plane case reduce to only seven:

$$
\begin{aligned}
E_{1121}= & E_{1112}, E_{1221}=E_{1212}, E_{2111}=E_{1211}, E_{2112}=E_{1212}, \\
& E_{2121}=E_{2112}, E_{2122}=E_{1222}, E_{2221}=E_{2212}
\end{aligned}
$$

- 15 major symmetries, consequences of the existence of an elastic potential $W=\frac{1}{2} \boldsymbol{\sigma} \cdot \boldsymbol{\varepsilon}: \sigma_{i j}=\frac{\partial W}{\partial \varepsilon_{i j}} \Rightarrow$

$$
E_{i j k l}=E_{k l i j},
$$

that for the plane case reduce to only three:

$$
E_{1211}=E_{1112}, E_{2211}=E_{1122}, E_{2212}=E_{1222} .
$$

When a tensor has only these index symmetries, it describes a so-called multi-constant material: for the complete anisotropic case it depends upon only 18 tensor invariants, plus 3 frame-dependent quantities, for a whole of 21 independent components. In the plane case, there are 5 invariants plus a quantity taking into account for the frame orientation. In both the cases, the isotropic case reduces to two distinct invariants or some of their functions, like the Young's modulus and the Poisson's coefficient, or the bulk and shear moduli or the Lamé's coefficients etc.

For the so-called rari-constant materials, the behavior is described by only 12 tensor invariants plus 3 quantities fixing the frame, for a whole of 15 independent components. For the plane case, we have 5 independent components, 4 of which are invariants, and isotropy is always described by a unique invariant quantity.

A classical result, is that for rari-constant materials $\mathbb{E}$ has 6 supplementary index symmetries, the so-called Cauchy-Poisson symmetries:

$$
E_{i j k l}=E_{i k j l},
$$


that for the plane case reduce to the only supplementary condition

$$
E_{1122}=E_{1212},
$$

which gives, actually, the reduction of the independent components from 6 to 5 .

In the following, we will identify rari-constant tensors with those satisfying the Cauchy-Poisson conditions, and concentrate exclusively on the plane case. We will see that identifying rari-constant materials is not so simple, because there are two possible and dual rari-constant materials, at least in $\mathbb{R}^{2}$.

Let us before introduce the major results of the polar formalism for representing tensors in $\mathbb{R}^{2}$ through invariants, Verchery [1979], Vannucci [2005]. Basically, then polar formalism introduces a complex variable transformation, the transformation of Verchery, that allows to obtain rotation matrices in $\mathbb{R}^{2}$ that are diagonal and symmetry matrices that are anti-diagonal. This fact renders the search of invariants and the analysis of elastic symmetries particularly simple. In the end, the Cartesian components of $\mathbb{E}$ are expressed as

$$
\begin{aligned}
& E_{1111}(\theta)=T_{0}+2 T_{1}+R_{0} \cos 4\left(\Phi_{0}-\theta\right)+4 R_{1} \cos 2\left(\Phi_{1}-\theta\right), \\
& E_{1112}(\theta)=R_{0} \sin 4\left(\Phi_{0}-\theta\right)+2 R_{1} \sin 2\left(\Phi_{1}-\theta\right), \\
& E_{1122}(\theta)=-T_{0}+2 T_{1}-R_{0} \cos 4\left(\Phi_{0}-\theta\right), \\
& E_{1212}(\theta)=T_{0}-R_{0} \cos 4\left(\Phi_{0}-\theta\right), \\
& E_{1222}(\theta)=-R_{0} \sin 4\left(\Phi_{0}-\theta\right)+2 R_{1} \sin 2\left(\Phi_{1}-\theta\right), \\
& E_{2222}(\theta)=T_{0}+2 T_{1}+R_{0} \cos 4\left(\Phi_{0}-\theta\right)-4 R_{1} \cos 2\left(\Phi_{1}-\theta\right) .
\end{aligned}
$$

The Cartesian components are hence functions of four moduli, $T_{0}, T_{1}, R_{0}$ and $R_{1}$, and of two angles, $\Phi_{0}$ and $\Phi_{1}$; the moduli and the angular difference $\Phi_{0}-\Phi_{1}$ constitute a complete set of independent invariants for $\mathbb{E}$. Fixing one of the two polar angles corresponds to fix a frame where the angle $\theta$ is measured. The converse of eqs. (7) are

$$
\begin{aligned}
& 8 T_{0}=E_{1111}(\theta)-2 E_{1122}(\theta)+4 E_{1212}(\theta)+E_{2222}(\theta) \\
& 8 T_{1}=E_{1111}(\theta)+2 E_{1122}(\theta)+E_{2222}(\theta), \\
& 8 R_{0} e^{4 i\left(\Phi_{0}-\theta\right)}=E_{1111}(\theta)-2 E_{1122}(\theta)-4 E_{1212}(\theta)+E_{2222}(\theta)+ \\
& \quad+4 i\left[E_{1112}(\theta)-E_{1222}(\theta)\right], \\
& 8 R_{1} e^{2 i\left(\Phi_{1}-\theta\right)}=E_{1111}(\theta)-E_{2222}(\theta)+2 i\left[E_{1112}(\theta)+E_{1222}(\theta)\right],
\end{aligned}
$$

It is apparent from the above equations that $T_{0}$ and $T_{1}$ are isotropy invariants, while $R_{0}, R_{1}$ and $\Phi_{0}-\Phi_{1}$ are anisotropy invariants. It can be shown that $T_{0}$ and $T_{1}$ are the only linear invariants of $\mathbb{E}$ while $R_{0}$ and $R_{1}$ are functions of quadratic invariants and $\Phi_{0}-\Phi_{1}$ of a cubic invariant, 
Vannucci [2005]. A linear invariant is necessarily a polynomial composed by the first powers of the Cartesian components of $\mathbb{E}$, while a quadratic one is a combination of the second powers and the cubic one of the third powers. It is, however, interesting to note that in the polar formalism the direct use of angles is possible just because the cubic invariant introduced is a function of a cubic invariant but not a polynomial one.

Equation (7) shows that anisotropy is the sum of two isotropic contributions, of course invariants, plus two anisotropic ones; these last are sinusoidal functions whose amplitudes are proportional to the anisotropic invariants $R_{0}$ and $R_{1}$ and that are shifted by an angle which is just equal to the last invariant: $\Phi_{0}-\Phi_{1}$.

It is also possible to express the components of the compliance tensor $\mathbb{S}$, the inverse of $\mathbb{E}$ : denoting the polar parameters of $\mathbb{S}$ by lower-case letters, it is

$$
\begin{aligned}
& S_{1111}(\theta)=t_{0}+2 t_{1}+r_{0} \cos 4\left(\varphi_{0}-\theta\right)+4 r_{1} \cos 2\left(\varphi_{1}-\theta\right), \\
& S_{1112}(\theta)=r_{0} \sin 4\left(\varphi_{0}-\theta\right)+2 r_{1} \sin 2\left(\varphi_{1}-\theta\right), \\
& S_{1122}(\theta)=-t_{0}+2 t_{1}-r_{0} \cos 4\left(\varphi_{0}-\theta\right), \\
& S_{1212}(\theta)=t_{0}-r_{0} \cos 4\left(\varphi_{0}-\theta\right), \\
& S_{1222}(\theta)=-r_{0} \sin 4\left(\varphi_{0}-\theta\right)+2 r_{1} \sin 2\left(\varphi_{1}-\theta\right), \\
& S_{2222}(\theta)=t_{0}+2 t_{1}+r_{0} \cos 4\left(\varphi_{0}-\theta\right)-4 r_{1} \cos 2\left(\varphi_{1}-\theta\right) .
\end{aligned}
$$

with

$$
\begin{aligned}
& t_{0}=\frac{2}{\Delta}\left(T_{0} T_{1}-R_{1}^{2}\right), \\
& t_{1}=\frac{1}{2 \Delta}\left(T_{0}^{2}-R_{0}^{2}\right), \\
& r_{0} e^{4 i \varphi_{0}}=\frac{2}{\Delta}\left(R_{1}^{2} e^{4 i \Phi_{1}}-T_{1} R_{0} e^{4 i \Phi_{0}}\right), \\
& r_{1} e^{2 i \varphi_{1}}=-\frac{R_{1} e^{2 i \Phi_{1}}}{\Delta}\left[T_{0}-R_{0} e^{4 i\left(\Phi_{0}-\Phi_{1}\right)}\right] .
\end{aligned}
$$

where

$$
\Delta=8 T_{1}\left(T_{0}^{2}-R_{0}^{2}\right)-16 R_{1}^{2}\left[T_{0}-R_{0} \cos 4\left(\Phi_{0}-\Phi_{1}\right)\right] .
$$

\section{Rari-constant elastic tensors}

We can easily state now the algebraic conditions for the elastic tensor $\mathbb{E}$ in $\mathbb{R}^{2}$ to be rari-constant:

Theorem 1. $\mathbb{E}$ is a rari-constant elastic tensor in $\mathbb{R}^{2} \Longleftrightarrow T_{0}=T_{1}$.

Proof. The proof is immediate: if $\mathbb{E}$ is a rari-constant tensor, then $E_{1212}(\theta)=$ $E_{1122}(\theta) \forall \theta$, and eqs. $\left(7_{3,4}\right)$ give $T_{0}=T_{1}$. Conversely, if $T_{0}=T_{1}$, then eqs. $\left(8_{1,2}\right)$ give $E_{1212}(\theta)=E_{1122}(\theta) \forall \theta$. 
Let us consider all the consequences of such a result:

- the number of independent tensor invariants is linked to the number of index symmetries, see also Vannucci [2010b]; in particular, a supplementary index symmetry corresponds to the identity of two invariants, so that the number of independent invariants is decreased by one;

- the rari-constant condition affects only the isotropic part of $\mathbb{E}$, i.e. only its linear invariants: the anisotropic part is not touched by the Cauchy-Poisson conditions, so that multi- and rari-constant materials share all the same types of elastic symmetries;

- the bounds on the polar parameters, see Vannucci and Desmorat [2015], consequence of the positive definiteness of $\mathbb{E}$,

$$
\begin{aligned}
& T_{0}-R_{0}>0, \\
& T_{1}\left(T_{0}^{2}-R_{0}^{2}\right)-2 R_{1}^{2}\left[T_{0}-R_{0} \cos 4\left(\Phi_{0}-\Phi_{1}\right)\right]>0, \\
& R_{0} \geq 0 \\
& R_{1} \geq 0
\end{aligned}
$$

do not exclude the existence of the case $T_{0}=T_{1}$ : in the classical frame of continuum elastic bodies, materials with a rari-constant tensor $\mathbb{E}$ are possible;

- the existence of multi-constant materials with $T_{0}=T_{1}$ is not allowed; this point is essential: apparently, just because eqs. (12) do not exclude the case $T_{0}=T_{1}$ for multi-constant materials, then such materials could exist; nevertheless, this is not possible, because of Theorem 1 ; physically, this means that whenever $T_{0}=T_{1}$, then tensor $\mathbb{E}$ is necessarily rari-constant: $E_{1212}(\theta)=E_{1122}(\theta) \forall \theta$ : a particular value of the tensor invariants determine a change of the algebraic structure of the elastic tensor;

A fundamental remark can now be done: all what has been said for $\mathbb{E}$ is equally valid for $\mathbb{S}$ : we can define a dual class of rari-constant materials, where the Cauchy-Poisson conditions are valid for the compliance tensor $\mathbb{S}$. We name in the following direct- and inverse- rari-constant materials those for which the Cauchy-Poisson condition (6) holds respectively for $\mathbb{E}$ or for $\mathbb{S}$. These two classes are necessarily distinct, i.e. it cannot exist a material being at the same time direct- and inverse- rari-constant: the Cauchy-Poisson conditions cannot be satisfied at the same time by $\mathbb{E}$ and $\mathbb{S}$. That is why the name rari-constant has been used not only to denote a class of materials, but also a type of elastic tensor: this distinction is necessary in the following.

Let us consider why a material cannot be at the same time direct- and rari-constant: 
Theorem 2. The Cauchy-Poisson condition (6) cannot be satisfied at the same time by $\mathbb{E}$ and $\mathbb{S}$.

Proof. Be $\mathbb{E}$ rari-constant, i.e. $E_{1122}=E_{1212}$; then $T_{0}=T_{1}$ by Theorem 1 . The polar invariants of $\mathbb{S}$ can then be calculated through eqs. (10) and (11) that in this case become:

$$
\begin{aligned}
& t_{0}=\frac{2}{\Delta}\left(T_{0}^{2}-R_{1}^{2}\right), \\
& t_{1}=\frac{1}{2 \Delta}\left(T_{0}^{2}-R_{0}^{2}\right), \\
& r_{0} e^{4 i \varphi_{0}}=\frac{2}{\Delta}\left(R_{1}^{2} e^{4 i \Phi_{1}}-T_{0} R_{0} e^{4 i \Phi_{0}}\right), \\
& r_{1} e^{2 i \varphi_{1}}=-\frac{R_{1} e^{2 i \Phi_{1}}}{\Delta}\left[T_{0}-R_{0} e^{4 i\left(\Phi_{0}-\Phi_{1}\right)}\right] .
\end{aligned}
$$

with

$$
\Delta=8 T_{0}\left(T_{0}^{2}-R_{0}^{2}\right)-16 R_{1}^{2}\left[T_{0}-R_{0} \cos 4\left(\Phi_{0}-\Phi_{1}\right)\right] .
$$

It is then apparent that

$$
t_{0}=t_{1} \Longleftrightarrow T_{0}^{2}=\frac{4 R_{1}^{2}-R_{0}^{2}}{3} .
$$

This value of $T_{0}$ is incompatible with the elastic bounds (12), see Appendix, and hence, $t_{0} \neq t_{1}$ when $T_{0}=T_{1}$, so by Theorem 1 applied to $\mathbb{S}, S_{1212} \neq$ $S_{1122}$.

The consequence is immediate: it is not correct to identify automatically rari-constant materials in $\mathbb{R}^{2}$ with the Cauchy-Poisson condition, because this concerns only one of the two elastic tensors of the material.

So, if $\mathbb{E}$ is rari-constant, it has only 5 distinct Cartesian components, but its inverse, $\mathbb{S}$ has 6 different components. Conversely, if $\mathbb{S}$ is rari-constant, it has 5 distinct Cartesian components, but they are 6 for $\mathbb{E}$. Nevertheless, in both the cases the number of independent tensor invariants is 4 . In fact, if $\mathbb{E}$ is rari-constant, then $T_{0}=T_{1}$ and by eqs. (13) we get

$$
t_{1}=\frac{T_{0}^{2}-R_{0}^{2}}{4\left(T_{0}^{2}-R_{1}^{2}\right)} t_{0} .
$$

Hence, though $t_{1} \neq t_{0}$, it is proportional to $t_{0}$. Of course, a similar relation exists for the dual case of $\mathbb{S}$ rari-constant, it is sufficient to swap lower- and upper-case letters.

Finally, there are two dual families of rari-constant materials: 
- the direct rari-constant materials:

$$
\begin{aligned}
& E_{1212}(\theta)=E_{1122}(\theta) \forall \theta, \\
& T_{0}=T_{1}, \\
& S_{1212}(\theta) \neq S_{1122}(\theta), \\
& t_{1}=\frac{T_{0}^{2}-R_{0}^{2}}{4\left(T_{0}^{2}-R_{1}^{2}\right)} t_{0},
\end{aligned}
$$

and

$$
\begin{aligned}
& E_{1111}(\theta)=3 T_{0}+R_{0} \cos 4\left(\Phi_{0}-\theta\right)+4 R_{1} \cos 2\left(\Phi_{1}-\theta\right), \\
& E_{1112}(\theta)=R_{0} \sin 4\left(\Phi_{0}-\theta\right)+2 R_{1} \sin 2\left(\Phi_{1}-\theta\right), \\
& E_{1122}(\theta)=E_{1212}(\theta)=T_{0}-R_{0} \cos 4\left(\Phi_{0}-\theta\right), \\
& E_{1222}(\theta)=-R_{0} \sin 4\left(\Phi_{0}-\theta\right)+2 R_{1} \sin 2\left(\Phi_{1}-\theta\right), \\
& E_{2222}(\theta)=3 T_{0}+R_{0} \cos 4\left(\Phi_{0}-\theta\right)-4 R_{1} \cos 2\left(\Phi_{1}-\theta\right) .
\end{aligned}
$$

- the inverse rari-constant materials:

$$
\begin{aligned}
& S_{1212}(\theta)=S_{1122}(\theta) \forall \theta, \\
& t_{0}=t_{1}, \\
& E_{1212}(\theta) \neq E_{1122}(\theta) \forall \theta, \\
& T_{1}=\frac{t_{0}^{2}-r_{0}^{2}}{4\left(t_{0}^{2}-r_{1}^{2}\right)} T_{0},
\end{aligned}
$$

and

$$
\begin{aligned}
& S_{1111}(\theta)=3 t_{0}+r_{0} \cos 4\left(\varphi_{0}-\theta\right)+4 r_{1} \cos 2\left(\varphi_{1}-\theta\right), \\
& S_{1112}(\theta)=r_{0} \sin 4\left(\varphi_{0}-\theta\right)+2 r_{1} \sin 2\left(\varphi_{1}-\theta\right), \\
& S_{1122}(\theta)=S_{1212}(\theta)=t_{0}-r_{0} \cos 4\left(\varphi_{0}-\theta\right), \\
& S_{1222}(\theta)=-r_{0} \sin 4\left(\varphi_{0}-\theta\right)+2 r_{1} \sin 2\left(\varphi_{1}-\theta\right), \\
& S_{2222}(\theta)=3 t_{0}+r_{0} \cos 4\left(\varphi_{0}-\theta\right)-4 r_{1} \cos 2\left(\varphi_{1}-\theta\right) .
\end{aligned}
$$

A further consideration concerns strain energy, $V$ : a general result, Vannucci [2005], of the polar formalism is that

$$
\begin{aligned}
V & =\frac{1}{2} \varepsilon \cdot \mathbb{E} \varepsilon=4 T_{1} t^{2}+8 R_{1} \cos 2\left(\Phi_{1}-\varphi\right) r t+ \\
& +2\left[T_{0}+R_{0} \cos 4\left(\Phi_{0}-\varphi\right)\right] r^{2}
\end{aligned}
$$

with $t, r$ and $\varphi$ the polar components of the strain tensor:

$$
\varepsilon_{11}=t+r \cos 2 \varphi, \varepsilon_{22}=t-r \cos 2 \varphi, \varepsilon_{12}=r \sin 2 \varphi .
$$

Hence, for direct- rari-constant materials it is

$$
V=4 T_{0} t^{2}+8 R_{1} \cos 2\left(\Phi_{1}-\varphi\right) r t+2\left[T_{0}+R_{0} \cos 4\left(\Phi_{0}-\varphi\right)\right] r^{2},
$$


while for the inverse- ones

$$
V=4 \alpha T_{0} t^{2}+8 R_{1} \cos 2\left(\Phi_{1}-\varphi\right) r t+2\left[T_{0}+R_{0} \cos 4\left(\Phi_{0}-\varphi\right)\right] r^{2},
$$

where

$$
\alpha=\frac{t_{0}^{2}-r_{0}^{2}}{4\left(t_{0}^{2}-r_{1}^{2}\right)} .
$$

Finally, if we consider that special orthotropies, Vannucci [2002, 2005], are characterized by the vanishing of a tensor invariant, i.e. $R_{0}=0$ for the case of $R_{0}$-orthotropy, while $R_{1}=0$ for square-symmetry, or by being an invariant a function of the other ones, for the case of $r_{0}$-orthotropic materials, Vannucci [2010a], then it is clear that the only necessary and sufficient condition for identifying a rari-constant material, regardless of its type, i.e. independently of the number of distinct Cartesian components for $\mathbb{E}$ or $\mathbb{S}$, is that the number of independent linear tensor invariants must be one.

\section{Rari-constant anisotropic layers}

We show in this Section that it is actually possible to fabricate both the cases of direct- and inverse- rari-constant layers. This can be done using appropriate volume fractions of unidirectional fibers to reinforce an isotropic matrix. Just as an example, rather simple and having the only purpose to show that these materials can be designed, we use the classical technical laws of homogenization, Jones [1999] ( $E$ indicates a Young's modulus, $G$ a shear modulus and $\nu$ a Poisson's coefficient):

$$
\begin{aligned}
& E_{1}=v_{f} E_{f}+\left(1-v_{f}\right) E_{m}, \\
& E_{2}=\frac{E_{f} E_{m}}{\left(1-v_{f}\right) E_{f}+v_{f} E_{m}}, \\
& G_{12}=\frac{G_{f} G_{m}}{\left(1-v_{f}\right) G_{f}+v_{f} G_{m}}, \\
& \nu_{12}=v_{f} \nu_{f}+\left(1-v_{f}\right) \nu_{m} .
\end{aligned}
$$

The above relations give the technical constants for an orthotropic layer reinforced by unidirectional fibers; the subscript $f$ denotes a constant of the fibers and $m$ of the matrix; $v_{f}$ is the volume fraction of the fibers, $0 \leq v_{f} \leq 1$.

We pose

$$
E_{f}=m E_{m}, \quad \nu_{f}=n \nu_{m},
$$

and remark that usually $m \gg 1$ while $n \sim 1$. Then, remembering that for the isotropic case (so are assumed to be the fibers and the matrix)

$$
G=\frac{E}{2(1+\nu)},
$$


we obtain

$$
\begin{aligned}
& E_{1}=\left[1+(m-1) v_{f}\right] E_{m}, \\
& E_{2}=\frac{m E_{m}}{m+v_{f}(1-m)}, \\
& G_{12}=\frac{m E_{m}}{2\left[m\left(1-v_{f}\right)\left(1+\nu_{m}\right)+v_{f}\left(1+n \nu_{m}\right)\right]}, \\
& \nu_{12}=\left[1+(n-1) v_{f}\right] \nu_{m} .
\end{aligned}
$$

Then, a classical result for an orthotropic layer, Jones [1999], is that

$$
\begin{aligned}
& E_{1111}=\frac{E_{1}}{1-\nu_{12} \nu_{21}}, \\
& E_{2222}=\frac{E_{2}}{1-\nu_{12} \nu_{21}}, \\
& E_{1122}=\frac{\nu_{12} E_{2}}{1-\nu_{12} \nu_{21}}, \\
& E_{1212}=G_{12}, \\
& E_{1112}=E_{1222}=0,
\end{aligned}
$$

and

$$
\nu_{21}=\nu_{12} \frac{E_{2}}{E_{1}} .
$$

Finally, one should remember eqs. $\left(8_{1,2}\right)$ :

$$
\begin{aligned}
& 8 T_{0}=E_{1111}-2 E_{1122}+4 E_{1212}+E_{2222} \\
& 8 T_{1}=E_{1111}+2 E_{1122}+E_{2222}
\end{aligned}
$$

Then, let us now consider the direct- rari-constant case: the condition to be satisfied is

$$
T_{0}=T_{1},
$$

completely equivalent to the Cauchy-Poisson condition

$$
E_{1212}=E_{1122} .
$$

Injecting successively eq. (29) into eq. (30) and then into eq. (32), after some standard passages condition (33) becomes

$$
\begin{aligned}
& {\left[1+(m-1) v_{f}\right]\left[m+v_{f}(1-m)\right]-m \nu_{m}^{2}\left[1+(n-1) v_{f}\right]^{2}-} \\
& 2 \nu_{m}\left[1+(m-1) v_{f}\right]\left[1+(n-1) v_{f}\right]\left[m\left(1-v_{f}\right)\left(1+\nu_{m}\right)+v_{f}\left(1+n \nu_{m}\right)\right]=0 .
\end{aligned}
$$

The only material parameter entering the above condition is $\nu_{m}$; this condition depends upon $m, n$ and $v_{f}$. The solution of such equation, for different 

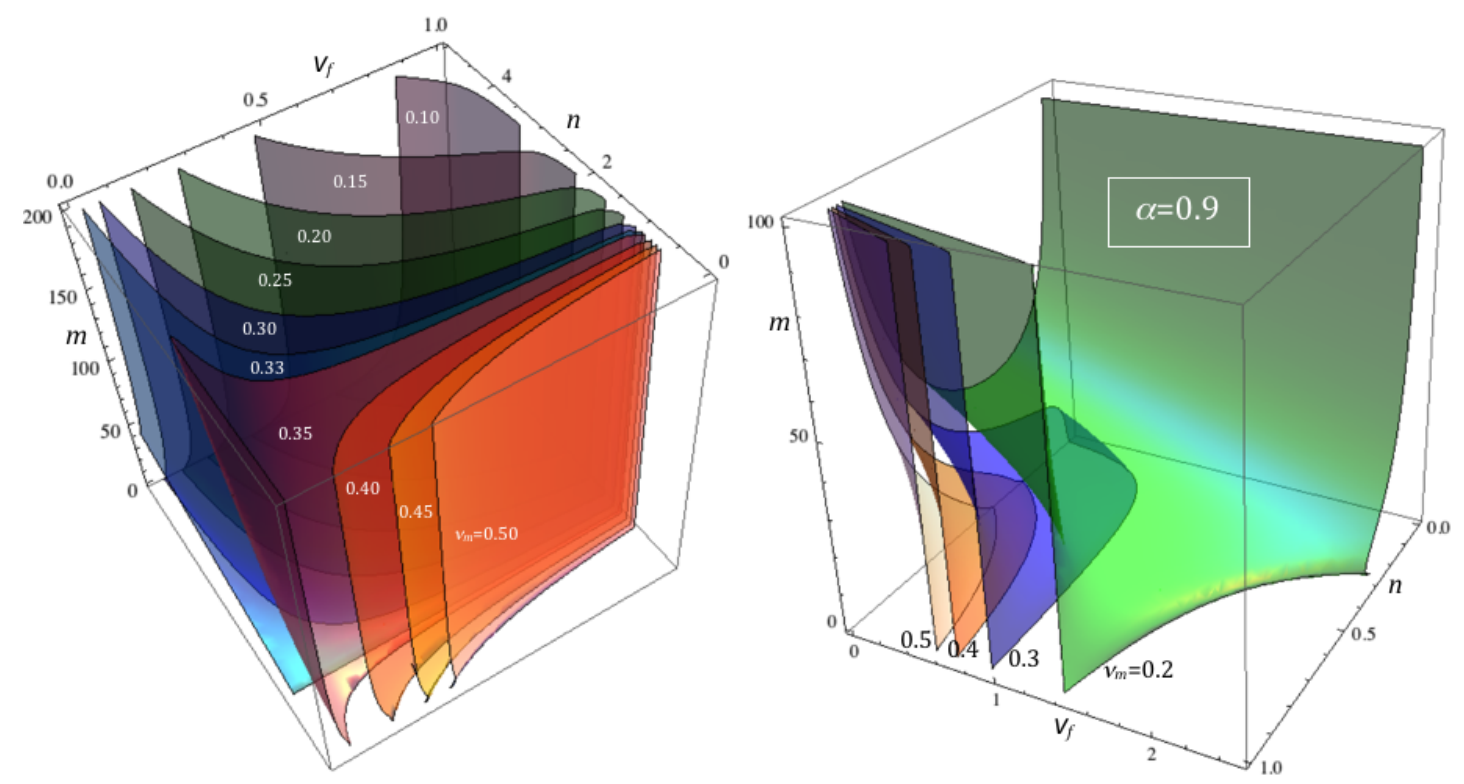

Figure 1: Solutions for rari-constant layers: the direct- and inverse- cases.

values of $\nu_{m}$, is plotted in the left diagram of Fig. 1. For a given couple of materials, fibers and matrix, $m$ and $n$ are fixed and eq. (35) allows for finding a value of the volume fraction of fibers $v_{f}$ resulting in a directrari-constant layer.

The inverse- rari-constant case is characterized by

$$
t_{0}=t_{1},
$$

which gives, for the stiffness constant, the condition

$$
T_{1}=\alpha T_{0}
$$

with $\alpha$ given by eq. (25). The passage to the stiffness components is necessary because the homogenization formulae (26) are given as functions of these ones. Unlike the previous case of direct- rari-constant layers, now condition (37) is not equivalent to the Cauchy-Poisson symmetry condition, but to the following one, eq. (32),

$$
\left(E_{1111}+E_{2222}\right)(1-\alpha)+2 E_{1122}(1+\alpha)-4 \alpha E_{1212}=0,
$$

which becomes, after introducing eqs. (29), (30) and some standard pas- 
sages,

$$
\begin{aligned}
& {\left[1+(m-1) v_{f}\right]\left\{\left[(m-1)^{2} v_{f}^{2}-(m-1)^{2} v_{f}-2 m\right](\alpha-1)+\right.} \\
& \left.2 m(\alpha+1)\left[1+v_{f}(n-1)\right] \nu_{m}\right\}\left[v_{f}\left(1+n \nu_{m}\right)+m\left(1-v_{f}\right)\left(1+\nu_{m}\right)\right]+ \\
& 2 m \alpha\left\{\left(v_{f}-1\right) v_{f}+m^{2}\left(v_{f}-1\right) v_{f}+\right. \\
& \left.m\left[\left[1+v_{f}(n-1)\right]^{2} \nu_{m}^{2}\right]-2 v_{f}^{2}+2 v_{f}-1\right\}=0 .
\end{aligned}
$$

In this case, the solution depends also upon the parameter $\alpha$, i.e. upon the compliance invariants, those of $\mathbb{S}$, of the final layer. These can be calculated only inverting the stiffness tensor $\mathbb{E}$, so the problem is nonlinear and the computation can be done iterating. Anyway, like in the case of the directrari-consant layers, also in this case it is possible to find from eq. (39) a value of the volume fraction of the fibers $v_{f}$ to obtain an inverse- rari-constant layer; eq. (39) is of the fourth degree in $v_{f}$, hence a numerical procedure is needed for the resolution. The solution of eq. (39) is plotted in the right diagram of Fig. 1.

A question concerns $\alpha$ : can $\alpha$ take any value? Actually not, and this can be seen in the following way: introduce the two dimensionless parameters

$$
\eta_{0}=\frac{r_{0}}{t_{0}}, \eta_{1}=\frac{r_{1}}{t_{0}} .
$$

Then,

$$
\alpha=\frac{1-\eta_{0}^{2}}{4\left(1-\eta_{1}^{2}\right)},
$$

and we can study its variation as a function of $\eta_{0}$ and $\eta_{1}$, considering that the bounds (12) applied to an orthotropic $\mathbb{S}$ and for $t_{0}=t_{1}$ give

$$
\begin{aligned}
& 0 \leq \eta_{0}<1, \\
& 0 \leq \eta_{1}<\sqrt{\frac{1+(-1)^{k} \eta_{0}}{2}} .
\end{aligned}
$$

Function $\alpha\left(\eta_{0}, \eta_{1}\right)$ is plotted in Fig. 2 and it is evident that $0<\alpha<1$. Incidentally, the fact that for inverse- rari-constant layers it cannot be $\alpha=1$ confirms that a material cannot be at the same time direct- and inverserari-constant.

\section{The isotropic case}

Two isotropic rari-constant materials can exist, the direct- and the inverseone. Let us briefly consider their properties. 


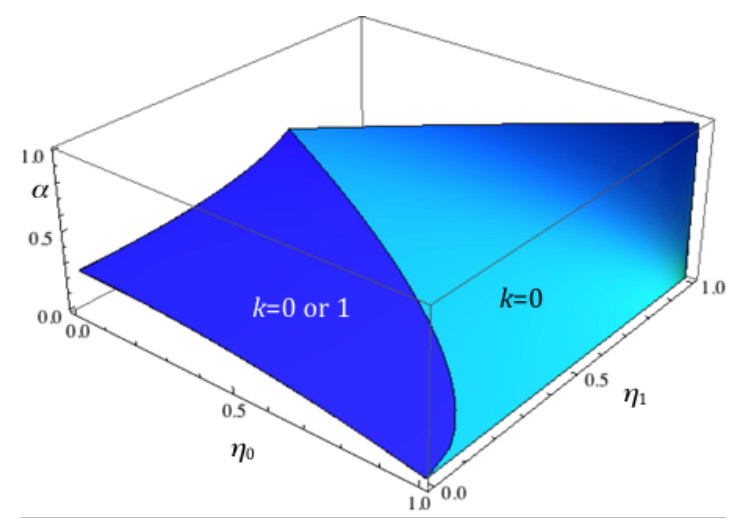

Figure 2: Function $\alpha\left(\eta_{0}, \eta_{1}\right)$.

The direct case first: isotropy is characterized by the vanishing of the anisotropic part, i.e. by

$$
R_{0}=R_{1}=0 \Rightarrow r_{0}=r_{1}=0
$$

The stiffness behavior is uniquely determined by $T_{0}$ :

$$
E_{1111}(\theta)=E_{2222}(\theta)=3 T_{0}, E_{1122}(\theta)=T_{0}, E_{1112}(\theta)=E_{1222}(\theta)=0 \forall \theta .
$$

For $\mathbb{S}$, it is

$$
t_{0}=\frac{1}{4 T_{0}}, t_{1}=\frac{1}{16 T_{0}} \Rightarrow t_{0}=4 t_{1}
$$

and, $\forall \theta$,

$$
\begin{aligned}
& S_{1111}(\theta)=S_{2222}(\theta)=t_{0}+2 t_{1}=\frac{3}{2} t_{0}=\frac{3}{8 T_{0}}, \\
& S_{1122}(\theta)=-t_{0}+2 t_{1}=-\frac{t_{0}}{2}=-\frac{1}{8 T_{0}}, \\
& S_{1212}(\theta)=t_{0}=\frac{1}{4 T_{0}} \Rightarrow S_{1212}(\theta)=-2 S_{1122}(\theta), \\
& S_{1112}(\theta)=S_{1222}(\theta)=0 .
\end{aligned}
$$

We can also introduce the classic technical constants:

$$
\begin{aligned}
& E:=\frac{1}{S_{1111}}=\frac{8}{3} T_{0}, \nu:=-\frac{S_{1122}}{S_{1111}}=\frac{1}{3} \\
& G:=\frac{1}{4 S_{1212}}=T_{0}, \kappa:=\frac{1}{S_{1111}+2 S_{1122}+S_{2222}}=2 T_{0} .
\end{aligned}
$$

It is then apparent the mechanical meaning of $T_{0}$ : it is equal to the shear modulus $G$ for the isotropic case; the result for the Poisson's coefficient is 
also classical, but it is worth to remark that it is only a necessary but not sufficient for a material to be direct- rari-constant: multi-constant materials with $\nu=1 / 3$ do exist. Also, for these materials the bulk modulus $\kappa$ is twice the shear modulus: they have a stiffness to spherical stress states that is the double of that to shear states. For the normal stiffness, this is $8 / 3$ times the shear one.

Finally, for what concerns the Lamé's constants, it is

$$
\lambda:=\kappa-G=T_{0}, \mu:=G=T_{0} \Rightarrow \lambda=\mu,
$$

a classical result.

Let us now turn the attention to inverse- rari-constant materials; now, $t_{0}$ uniquely determines all the distinct components of $\mathbb{S}$ :

$$
S_{1111}(\theta)=S_{2222}(\theta)=3 t_{0}, S_{1122}(\theta)=t_{0}, S_{1112}(\theta)=S_{1222}(\theta)=0 \forall \theta .
$$

For tensor $\mathbb{E}$, we get

$$
T_{0}=\frac{1}{4 t_{0}}, T_{1}=\frac{1}{16 t_{0}} \Rightarrow T_{0}=4 T_{1}
$$

and, $\forall \theta$,

$$
\begin{aligned}
& E_{1111}(\theta)=E_{2222}(\theta)=T_{0}+2 T_{1}=\frac{3}{2} T_{0}=\frac{3}{8 t_{0}}, \\
& E_{1122}(\theta)=-T_{0}+2 T_{1}=-\frac{T_{0}}{2}=-\frac{1}{8 t_{0}}, \\
& E_{1212}(\theta)=T_{0}=\frac{1}{4 t_{0}} \Rightarrow E_{1212}(\theta)=-2 E_{1122}(\theta), \\
& E_{1112}(\theta)=E_{1222}(\theta)=0 .
\end{aligned}
$$

Now, the technical constants are

$$
E=\frac{4}{3} T_{0}, \nu=-\frac{1}{3}, G=T_{0}, \kappa=\frac{T_{0}}{2} .
$$

Inverse- rari-constant materials are hence necessarily materials with a negative Poisson's coefficient, whose value is exactly the opposite of the direct case; nevertheless, they can exist. Also, their normal stiffness is just half and their bulk modulus a fourth of the corresponding direct case ones. Now, the spherical stiffness is smaller than the shear one.

The Lamé's constants now are

$$
\lambda=-\frac{T_{0}}{2}, \mu=T_{0} \Rightarrow \lambda=-\frac{\mu}{2},
$$

i.e. $\lambda$ is negative; nevertheless, thanks to eq. (12), the bounds on the values of the Lamé's constants in $\mathbb{R}^{2}$ are satisfied:

$$
\mu=T_{0}>0, \lambda+\mu=\frac{T_{0}}{2}>0 .
$$


Concerning the strain energy, eq. (23) becomes

$$
V=2 T_{0}\left(2 t^{2}+r^{2}\right)
$$

while eq. (24), being for the isotropic case $\alpha=1 / 4$, becomes

$$
V=T_{0}\left(t^{2}+2 r^{2}\right)
$$

It is interesting to notice, as easily seen from eq. (22), that, if we indicate by $V_{s p h}$ and $V_{\text {dev }}$ respectively the spherical and deviatoric part of $V$, then $V_{s p h}$ is proportional to $t^{2}$ and $V_{\text {dev }}$ to $r^{2}$, so that $V_{s p h} \sim 2 V_{\text {dev }}$ for the directcase while $V_{s p h} \sim V_{d e v} / 2$ for the inverse- one. In other words, direct- rariconstant materials stock better the elastic energy under the form of energy linked to volume changes than to shape changes, while for the inverse- ones it is exactly the opposite.

\section{Final considerations}

An old question, the existence and properties of rari-constant materials, has been approached in a new way, the polar method for plane anisotropy, in the framework of continuum elasticity.

Some points have been clearly shown:

- there are exactly two dual classes of rari-constant materials, the directand inverse- types;

- the classical Cauchy-Poisson relations (actually, only one in $\mathbb{R}^{2}$ ) characterize only the algebraic properties of a tensor, not of a material;

- the necessary and sufficient condition for a material to be of the rariconstant type in $\mathbb{R}^{2}$ is that the number of independent linear, i.e. isotropic, invariants is one;

- rari-constant materials can actually exist, while multi-constant materials with $T_{0}=T_{1}$ or $t_{0}=t_{1}$ are not allowed;

- in the isotropic case, direct- rari-constant materials correspond to a material with a shear modulus half the bulk one, $G=\kappa / 2$, and a Poisson's coefficient $\nu=1 / 3$, while the inverse- ones to a material with $G=2 \kappa$ and to $\nu=-1 / 3$.

A final remark: the condition $T_{0}=T_{1}$ clearly indicates that the anisotropic part of a plane elastic tensor is necessarily rari-constant; in other words, in $\mathbb{R}^{2}$ only the isotropic part is responsible for the multi-constant behavior. 


\section{Appendix}

We show that the value

$$
T_{0}=\frac{4 R_{1}^{2}-R_{0}^{2}}{3}
$$

is incompatible with the elastic bounds (12) on the polar invariants for direct- rari-constant materials, i.e. when $T_{1}=T_{0}$.

Replacing eq. (57) into eq. (12 1 ) and taking into account for eqs. (12,4) gives

$$
R_{1}>R_{0}>0 .
$$

Now, injecting eq. (57) into eq. $\left(12_{2}\right)$ we get, after posing

$$
\begin{gathered}
\rho=\frac{R_{0}}{R_{1}}, \quad C=\cos 4\left(\Phi_{0}-\Phi_{1}\right), \quad 0 \leq \rho<1,-1 \leq C \leq 1, \\
\sqrt{\frac{4-\rho^{2}}{3}}<\frac{3 \rho C}{1+2 \rho^{2}},
\end{gathered}
$$

a condition that is satisfied if and only if

$$
\left\{\begin{array}{l}
\frac{4-\rho^{2}}{3} \geq 0 \\
\frac{3 \rho C}{1+2 \rho^{2}} \geq 0 \\
\frac{4-\rho^{2}}{3}<\frac{9 \rho^{2} C^{2}}{\left(1+2 \rho^{2}\right)^{2}} .
\end{array}\right.
$$

Condition (61 1 ) gives $\rho \leq 2$, which is redundant because of eq. (593), condition $\left(61_{2}\right)$ limits eq. $\left(59_{4}\right)$ to $0 \leq C \leq 1$ while condition $\left(61_{3}\right)$ can be rewritten as

$$
f=\frac{\left(4-\rho^{2}\right)\left(1+2 \rho^{2}\right)^{2}}{27 \rho^{2}}<C^{2},
$$

which is never satisfied because $f>1=\max C^{2}$ for $0 \leq \rho<1$, as it can be easily recognized.

The isotropic case is trivial, for eq. (57) should give $T_{0}=0$ which corresponds to a material with a null stiffness, hence it is impossible.

The two cases of special orthotropies are also impossible; in fact, the case of square symmetry, $R_{1}=0$, should imply a negative value for $T_{0}^{2}$, eq. (57), while that of $R_{0}$-orthotropy, Vannucci [2002], $R_{0}=0 \Rightarrow \rho=0$, gives $f \rightarrow \infty$. 


\section{References}

E. Benvenuto. An introduction to the history of structural mechanics. 2 vols. Springer Verlag, Berlin, 1991.

M. Born. Dynamik der Krystallgitter. Leipzig und Berlin, 1915.

R. G. Boscovich. Theoria philosophiae naturalis redacta ad unicam legem virium in natura existentium. Venice, 1743.

D. Capecchi, G. Ruta, and P. Trovalusci. Voigt and Poincaré's mechanisticenergetic approaches to linear elasticity and suggestions for multiscale modelling. Archive of Applied Mechanics, 81:1573-1584, 2011.

A. L. Cauchy. Sur l'équilibre et le mouvement d'un système de points matériels sollicités par des forces d'attraction ou de repulsion mutuelle. Exercices de mathématiques, 3:188-212, 1828a.

A. L. Cauchy. De la pression ou tension dans un système de points matériels. Exercices de mathématiques, pages 213-236, 1828b.

A. Barré de Saint-Venant. Sur la question de savoir s'il existe des masses continues et sur la nature probable des dernières particules des corps. Société Philomatique de Paris, pages 3-15, 1844.

T. C. Doyle and J. L. Ericksen. Nonlinear elasticity. Advances in applied Mechanics, 4:53-115, 1956.

R. Dugas. Histoire de la mécanique. Editions du Griffon, Neuchâtel, 1950.

G. Green. On the laws of refelxion and refraction of light at the common surface of two non-crystallized media. Cambridge Philosophical Society Transactions, 7, 1839.

R. M. Jones. Mechanics of composite materials - 2nd Edition. Taylor and Francis, Philadelphia, 1999.

A. E. H. Love. A treatise on the mathematical theory of elasticity. Dover, New York, 1944.

N. I. Muskhelishvili. Some basic problems of the mathematical theory of elasticity. Noordhoff, Groningen, 1953.

L. Navier. Mémoire sur les lois de l'équilibre et du mouvement des solides élastiques. Mémoires de l'Académie Royale des Sciences de l'Institut National, 7:375-393, 1827.

I. Newton. Philosophiae Naturalis Principia Mathematica. London, 1687. 
M. Ostoja-Starzewski. Microstructural Randomness and Scaling in Mechanics of Materials. Chapmann and Hall/CRC, New York, 2007.

H. Poincaré. Leçons sur la théorie de l'élasticité. Carré, Paris, 1892.

S. D. Poisson. Traité de mécanique. Paris, 1811.

S. D. Poisson. Mémoire sur les surfaces élastiques. Mémoires de l'Académie Royale des Sciences de l'Institut National, pages 167-225, 1814.

S. D. Poisson. Mémoire sur l'équiibre et le mouvement des corps élastiques. Mémoires de l'Académie Royale des Sciences de l'Institut National, 8: 357-570, 1829.

I. Stackgold. The cauchy relations in a molecular theory of elasticity. Quarterly of Applied Mathematics, 8:169-186, 1950.

I. Todhunter and K. Pearson. History of the theory of elasticity, vol. 1. Cambridge University Press, Cambridge, 1886.

P. Vannucci. A special planar orthotropic material. Journal of Elasticity, $67: 81-96,2002$.

P. Vannucci. Plane anisotropy by the polar method. Meccanica, 40:437-454, 2005.

P. Vannucci. On special orthotropy of paper. Journal of Elasticity, 99:75-83, 2010a. doi: 10.1007/s10659-009-9232-2.

P. Vannucci. Anisotropy of plane complex elastic bodies. International Journal of Solids and Structures, 47:1154-1166, 2010b.

P. Vannucci and B. Desmorat. Analytical bounds for damage induced planar anisotropy. International Journal of Solids and Structures, 2015. URL http://dx.doi.org/10.1016/j.ijsolstr.2015.02.017.

G. Verchery. Les invariants des tenseurs d'ordre 4 du type de l'élasticité. In Proc. of colloque Euromech 115, Villard-de-Lans, (France), 1979.

W. Voigt. Theoretische Studien über die Elasticitätsverhältnisse der Kristalle. Abhandlungender Gesellschaftder Wissenschaften Zu Göttingen-Mathematische Classe, 34:1-100, 1887. 